Les missions de productivité dans les années 1950 : une tentative pour importer en France une fonction d'expertise syndicale

French productivity missions in the USA in the 50': towards a trade-union expertise

\title{
Paula Cristofalo
}

\section{OpenEdition}

Journals

Édition électronique

URL : http://journals.openedition.org/travailemploi/4064

DOI : 10.4000/travailemploi.4064

ISSN : 1775-416X

Éditeur

DARES - Ministère du Travail

Édition imprimée

Date de publication : 5 décembre 2008

Pagination : 69-81

ISSN : 0224-4365

Référence électronique

Paula Cristofalo, «Les missions de productivité dans les années 1950 : une tentative pour importer en France une fonction d'expertise syndicale », Travail et Emploi [En ligne], 116 | octobre-décembre 2008, mis en ligne le 05 novembre 2010, consulté le 30 avril 2019. URL : http://journals.openedition.org/ travailemploi/4064; DOI : 10.4000/travailemploi.4064 


\title{
Les missions de productivité dans les années 1950: une tentative pour importer en France une fonction d'expertise syndicale
}

\author{
Paula Cristofalo (*)
}

\begin{abstract}
L'étude de quatre missions de productivité réalisées sous l'égide du ministère du Travail entre les années 1950 et 1953 et de la création des bureaux de recherche syndicaux qui les ont suivies permet d'étudier l'intervention de la direction du Travail en faveur des organisations syndicales dans le contexte de la politique de productivité. Tandis que les missions sont destinées à faire connaître le syndicalisme américain, ses modes de fonctionnement et par ce biais, à amener les syndicalistes français à s'approprier des questions jusqu'alors éloignées de leur pratique, les bureaux de recherche visent à promouvoir le développement d'une fonction technique syndicale et plus largement un autre type de relations professionnelles. Ces deux actions semblent être à l'origine de l'expertise syndicale telle qu'elle se pratique aujourd'hui en France.
\end{abstract}

Dans le contexte de l'après-guerre, une élite politico-administrative s'est emparée du diagnostic du retard français pour élaborer un programme d'action destiné à moderniser les structures économiques et sociales. Des fonctionnaires du ministère du Travail, et plus particulièrement de la direction du Travail, en accord avec des dirigeants d'entreprises et des représentants syndicaux, ont impulsé, grâce à l'aide financière américaine (plan Marshall, amendement Blair-Moody...), une série d'initiatives visant à changer les modes d'action des syndicats et, plus largement, à redéfinir leurs rôles dans les institutions.

Parmi ces actions figure l'organisation de «missions de productivité», voyages d'étude auxquels participent des représentants des organisations professionnelles. Divers travaux ont été menés sur l'impact de ces séjours à partir d'angles d'approche différents: leur fonction pédagogique (CROUzet, 2002), leur importance relative selon les secteurs économiques (BARJOT, 2002), leur influence sur l'importation du management (BolTANSKI, 1981, 1982) et sur le développement des sciences sociales du travail (TANGUY, 2008). D'autres ont plus largement souligné le changement de regard des missionnaires sur leur propre pays (GUIGUENo, 2002) et révélé, par contraste, ce qui manquait en France (KuISEL, 1996).

\footnotetext{
* Université de Paris X-Nanterre, Laboratoire genre, travail et mobilités (GTM). E-mail: paula.cristofalo@gmail.com
}

Parmi les 500 missions(1) réalisées entre les années 1949 et 1959, 55 l'ont été sous l'égide du ministère du Travail. Ces actions prennent place dans le cadre de la politique réformatrice impulsée par ce ministère (TANGUY, 2006), de l'instauration des plans de modernisation économique à la mise en place d'une politique de productivité que R. Boulat (2006; 2008) a minutieusement analysée. Paul Bacon, résistant, jociste, syndicaliste CFTC, député MRP, a occupé la fonction de ministre du Travail de manière quasi continue entre 1950 et 1962 . C'est dans son cabinet que la participation française à la productivité dans le plan Marshall est mise au point (BÉTHOUART, 1999; 2006). Des fonctionnaires de la direction du Travail tentent alors de profiter de cette entreprise pour provoquer des changements dans les relations professionnelles. À travers les missions de productivité et les dispositifs qui les prolongent, ils vont notamment essayer de compenser les faiblesses techniques des organisations syndicales en formant de véritables interlocuteurs syndicalistes, voire des experts. Sont impliqués dans cette politique, en première ligne, Édouard Lambert, inspecteur du travail, résistant, réputé pour " ses rapports excellents avec les organisations professionnelles et son tact» (BÉTHOUART, 2006: 240-241), membre du Conseil national de la productivité (CNP) et directeur du Travail entre 1948 et 1956, et Pierre Laurent, maître

(1) R. Kuisel (1988) compte 209 missions réalisées entre les années 1950 et 1952, période du plein essor de ces voyages; L. Boltanski (1982) dénombre quant à lui un total de 450 missions réalisées, et R. Boulat (2002) avance un nombre voisin de 500 missions. 
de requêtes au Conseil d'État et directeur général du Travail et de la main-d'œuvre entre 1957 et 1963. Au cours de ces années, comme l'affirme P. Fournier (Chatriot, 2006; Béthouart, 2006), rien d'important dans le domaine des relations professionnelles ne se fait sans l'approbation d'une figure majeure de ce ministère, Olga Raffalovich, résistante, femme d'action et après plusieurs activités dans ce ministère, sous-directrice des relations professionnelles (Maclouf, 2007). Betty Piguet, à l'instar d'autres femmes, souvent célibataires, qui occupent une place décisive au sein de ce ministère (СHATRIOT, 2006), travaille étroitement avec O. Raffalovich sur les questions syndicales et de représentation du personnel. Issue d'une famille protestante, diplômée en droit et ancienne de la Fédération des étudiants chrétiens (FEC), « $\mathrm{M}^{1 \mathrm{le}}{ }_{»}$ Piguet est considérée, grâce à sa compétence et son sens social élevé, comme l'un des meilleurs chefs de bureau de la direction (BÉTHOUART, 2006). Deux autres hauts fonctionnaires de cette sous-direction ont assuré la mise en place des actions en direction des organisations syndicales: C. Lapierre, J. Denoyelle. Enfin, on souligne le rôle joué par J. Gouin, inspecteur principal du travail et de la main-d'œuvre, représentant du ministère au CNP, chargé des aspects sociaux des programmes de productivité et qui a lui-même participé aux missions. Les archives de ce dernier(2) permettent de reconstituer les actions de ces fonctionnaires, leurs répercussions et leur portée.

Cet article se propose d'étudier quatre de ces missions ayant contribué à consolider l'enjeu de la fonction d'expertise comme une préoccupation partagée entre syndicalistes et hauts fonctionnaires. Ces missions, composées exclusivement de représentants des salariés, étaient destinées à faire connaître le syndicalisme américain et ses modes de fonctionnement. Il s'agissait, par ce biais, d'amener les syndicats français à s'approprier des questionnements jusqu'alors éloignés de leur pratique.

\section{Dépasser la méfiance et organiser un programme de missions}

$\mathrm{Si}$, au sortir de la guerre, les organisations syndicales sont convaincues de la nécessité de mener la «bataille» de la productivité, les points de vue divergent sur les modalités et les modes de participation (Fourastié et al., 1952). Cependant, dès la scission de la CGT (1947), dans un contexte marqué par la guerre froide, seule une partie du mouvement

(2) Archives conservées sous les cotes CAC 19760121, articles: 65 à $75,112,120$ à 125 et 128 . Une première version plus détaillée de ce texte a été publiée dans le rapport de recherche: L. Tanguy, L'institut des sciences sociales du travail. Contribution à l'histoire de la sociologie du travail en France 1950-1970, Dares, mars 2007, Chapitre III, pp. 70-113. ouvrier organisé(3) accepte de participer à la définition d'une politique de productivité. Il s'agit des trois confédérations syndicales de salariés dites «libres»: la CFTC, la CGT-FO et la CGC. Elles souhaitent répondre à la question technique essentielle du partage «équitable» des fruits de la productivité et du contrôle effectif des accords menés dans ce domaine, mais sous conditions. Elles demeuraient jusqu'alors relativement réservées, car des controverses étaient nées au sein de leurs propres structures et les frontières idéologiques entre confédérations rendaient l'entente encore plus délicate. Leur participation au programme de productivité résulte donc d'une série d'actions au cours desquelles elles nouent des compromis avec la direction du Travail et le CNP.

Ainsi, la CFTC déclare s'investir avec méfiance $\mathrm{du}$ fait que, dans de nombreuses situations, l'augmentation de la productivité ne s'est traduite ni par des augmentations de salaire ni par l'amélioration des conditions de vie des travailleurs. Elle entend donc conclure «un véritable contrat» qui garantisse son engagement par des avantages. Au préalable, elle devra convaincre ses adhérents de soutenir de telles activités et de coopérer avec les autres organisations syndicales (4). Elle bénéficiera de l'investissement dans cette tâche des responsables comme G. Levard (secrétaire général adjoint) et des contacts personnels qu'entretiennent quelques-uns de ses dirigeants avec des syndicalistes d'Outre-atlantique. Parmi ces dirigeants, le secrétaire général du Syndicat général de l'éducation nationale, P. Vignaux (5), entend exercer son influence au sein du ministère $\mathrm{du}$ Travail par l'intermédiaire de fonctionnaires rencontrés dans son parcours militant (JOC) et dans la résistance.

La CGT-FO, quant à elle, compte tenu de son histoire, s'engage d'emblée dans une coopération avec les autorités américaines. Outre l'appui financier apporté à cette confédération par des syndicats américains, la question d'une aide technique reste

(3) Les autorités américaines interdisent à la CGT d'y participer et, réciproquement, les cégétistes refusent d'y coopérer. (4) Des tendances internes s'opposent à une coopération avec la CGT-FO. Tel était le cas lorsque, face à l'opportunité de réaliser des sessions d'études communes financées par les fonds américains, un conflit interne a éclaté à ce propos (ADAM, 1964: 165)

(5) P. Vignaux (1904-1987), figure de référence des mouvements d'éducation ouvrière au sein de la CFTC, puis de la CFDT, est l'un des fondateurs du groupe Reconstruction, inspirateur du processus de déconfessionnalisation. Son rôle de médiateur entre les mouvements ouvriers français et américains pendant la guerre a été souvent mentionné (GoDSON, 1976; WALL, 1987) mais les effets ont été peu étudiés (JEANPIERRE, 2000: 55). L'arrivée de P. Bacon au ministère et, les contacts amicaux qu'il maintient avec certains de ses fonctionnaires lui permettent d'exercer pleinement ce rôle. $C f$. lettres de $\mathrm{P}$. Vignaux à J. Gouin. 
posée depuis son Congrès fondateur(6) mais la politique de productivité suscite l'opposition d'une partie des militants (7). Quelques dirigeants, comme $\mathrm{R}$. Bothereau qui défend une orientation réformiste (Pennetier, 2003 :41), comprennent que les salariés s'investissent faiblement, mais encouragent néanmoins les mesures qui renforcent les pouvoirs d'intervention des salariés au sein des entreprises. Les positions ne sont donc pas unanimes.

La CGC, elle, n'a que six années d'existence au moment où débute la politique de productivité. Elle entend y tenir un rôle actif, et ses responsables, à l'instar d'A. Malterre, voient dans cette politique une occasion d'affirmer la place de la confédération dans le panorama syndical et de compenser sa faible représentativité. Ces objectifs rencontrent ceux des fonctionnaires qui tentent d'intégrer cette confédération dans des actions où elle n'était originellement pas pressentie (8).

Pour participer au programme de productivité, ces trois organisations syndicales vont trouver des points de convergence, définissant leurs enjeux en termes techniques, délestés de toute référence aux positions de principe politiques.

La direction du Travail, pour sa part, défend l'idée que l'accroissement de la productivité peut contribuer au progrès social par une augmentation de la consommation et une meilleure répartition des richesses. Elle va surtout inscrire ses actions dans une volonté de pacification sociale en essayant d'amener les organisations syndicales à s'impliquer dans cette politique. C'est dans le cadre de ce programme que cette direction voit concrètement l'occasion d'agir en faveur d'une politique de participation des salariés dans l'entreprise. Pour cela, elle prend des initiatives afin de s'opposer aux arguments prétendant que la productivité contribue seulement à augmenter le profit du patronat et à imposer des cadences infernales aux salariés.

L'entente entre les confédérations syndicales, et entre celles-ci et les pouvoirs publics, se noue au cours d'un voyage «interprofessionnel» aux ÉtatsUnis en 1950. Il s'agit d'une mission «pilote» visant à étudier les conditions d'application de l'assistance technique américaine. Ce voyage semble être décisif dans le choix d'utilisation du principe des missions - déjà adopté par d'autres administrations - comme moyen d'action du ministère. Inspiré

(6) Lors de ce congrès, D. Williams, syndicaliste américain, est intervenu pour faire l'éloge de l'aide américaine présentée comme «un instrument qui aidera les ouvriers européens à reconquérir une sécurité économique et à atteindre un niveau de vie plus élevé». Compte rendu du congrès constitutif de la CGT-FO, Palais de la Mutualité, Paris, 13 avril 1948.

(7) $C f$. les comptes rendus des congrès confédéraux de la CGT-FO, notamment, à partir de 1952.

(8) En essayant, par exemple, de l'intégrer à certaines missions. d'une démarche anglo-saxonne(9) accordant le primat à l'expérience, il est perçu comme devant permettre aux représentants des organisations syndicales de prendre connaissance de la situation américaine, et de lever ainsi les méfiances et les craintes envers les résultats de la politique de productivité. Par la démarche comparative qu'ils impliquent, ces voyages devaient en outre induire(10) un effet de «retournement de miroir» mettant en évidence les aspects susceptibles d'être réformés.

Cette mission pilote est organisée par des membres de l'Economic Cooperation Administration (ECA) - P. Hoffman (administrateur du plan Marshall), W. Hoff (directeur de l'assistance technique de l'ECA), J. Silbermann (expert du gouvernement américain) et des dirigeants syndicaux américains, I. Abramson, J. Potofsky, D. Dubinsky.

L'équipe se compose de personnalités qui sont placées au cœur des processus de décision. Parmi celles-ci, deux représentants des organisations professionnelles patronales (11), neuf dirigeants des confédérations syndicales (12), le directeur de l'AFAP, P. Lemaresquier, un Inspecteur de l'Industrie, F. Chapel, et J. Gouin. Les représentants syndicaux sont des dirigeants capables de dégager des thèmes d'intérêt général, d'évaluer et de proposer le programme des missions à suivre, ainsi que de diffuser les idées retenues. Ils sont majoritairement des ingénieurs, partageant avec les hauts fonctionnaires des institutions de la productivité une formation scientifique et technique, ce qui leur permet de trouver plus aisément un langage commun (BOULAT, $2006: 421$ ).

Après avoir rencontré leurs homologues américains et visité près de vingt-cinq établissements, les participants exposent publiquement les principaux enseignements de la mission(13). Ils s'attachent à présenter la question de la productivité comme devant légitimement être posée par les organisations professionnelles et en concluent qu'en défini-

(9) Dans La productivité française $\left(\mathrm{n}^{\circ} 2\right.$, 1952) P. BIzE explique que cette démarche «solidement appuyée sur des habitudes américaines dont nous comprenions mal le sens il y a seulement deux ans [...] a un peu dérouté au début mais a rapidement prouvé sa valeur par ses résultats ».

(10) Tous les dossiers des missions organisées par ce ministère comportent une rubrique «justification de comparaison»" et il est toujours demandé que ce principe soit repris dans le rapport final.

(11) R. Christa et P. Vallée du CNPF.

(12) Pour la CFTC: A. Bapaume (vice-président), G. Esperet (secrétaire général adjoint) et G. Levard. Pour FO: J. Cucuel (commission exécutive) et deux secrétaires généraux, R. Richard (Fédération des ingénieurs et cadres) et J.-B. Tomas (EDFGDF). Enfin, les représentants de la CGC sont R. Speyser (viceprésident), J. Denolle (commission exécutive) et C. Boisumeau (secrétaire de la régie Renault).

(13) Bapaume, Chapel, Denolle, Gouin et Richard, «La productivité américaine», Les Echos, n ${ }^{\circ} 93,1950$. Actes de la conférence prononcée au Club Echos, le jeudi 14 décembre 1950. 
tive la concurrence est non seulement un moteur de la productivité aux États-Unis, mais aussi un facteur de progrès économique et social. Les analyses qu'ils retiennent de la productivité font cependant apparaître la diversité des grilles de lecture possibles: les représentants syndicaux placent logiquement au premier plan la question du pouvoir d'achat des salariés, alors que les représentants du CNPF mettent en avant le rôle du profit. Quoi qu'il en soit, les facteurs psychologiques et sociaux apparaissent aux yeux de la majorité des participants comme l'explication la plus marquante de la productivité américaine. Plus encore, l'information et la documentation spécifiques dont bénéficient les syndicalistes au cours des négociations sont jugées essentielles dans la construction de ce climat social. J. Gouin s'attache tout particulièrement à montrer l'importance de l'accès des salariés à l'information en matière de comptabilité, de prix de revient, etc.

Parallèlement à ce voyage, B. Bingham - le chef de mission de l'ECA - suggère à la direction du Travail l'envoi d'équipes supplémentaires, «au sein desquelles on réserverait une place particulièrement large aux militants des cercles syndicaux», et propose que ces équipes se consacrent à l'étude de «l'acceptation par les travailleurs de la productivité et des méthodes par lesquelles les syndicats et le patronat américain parviennent à obtenir cette acceptation » (14). La proposition insiste sur l'importance d'étudier plusieurs thèmes: la propagande auprès des ouvriers, la formation professionnelle, la recherche syndicale et la négociation collective. Les organisations syndicales sont alors une priorité dans le programme de l'ECA, pour qui «la France n'a pas encore réussi à créer un mouvement ouvrier puissant» (WALL, 1989: 261).

Les missions proposées font partie du programme général d'assistance technique (MCGLADE, 1995, 2002) dans lequel des responsables syndicaux américains sont engagés comme conseillers du travail pour l'ECA. Ces conseillers, tels que B. Shishkin, W. Green (Godson, $1976: 40$ ), et notamment les collaborateurs de l'administrateur P. Hoffmann: C. Golden et B. Jewell(15), ont pour tâche de prendre contact avec les syndicalistes français et de développer les missions de productivité (WALL, 1989).

Quant aux missions françaises composées exclusivement des représentants des salariés, elles semblent avoir tout d'abord privilégié une connaissance générale du mouvement ouvrier et du syndicalisme américains, avant que leur objet ne se déplace progressivement vers les méthodes d'action: la procédure, la formation, la comptabilité, les recherches syndicales

(14) Lettre datée du 2 juin 1950.

(15) Sur le rôle de ces syndicalistes-conseillers: $c f$. l'interview de B. Shishkin, «L'aide américaine à la France. Bulletin publiant des informations relatives au "Plan Marshall"», le 15 juillet 1948. et d'autres thèmes plus spécifiques présentés comme techniques(16). Désignées comme «missions sociales», elles ont une durée de six semaines. Chaque mission est composée, en moyenne, d'une douzaine de syndicalistes toujours accompagnés d'un représentant du ministère. Celui-ci, chef de mission, et «observateur impartial» connaissant bien la législation du travail, vise à «modérer la tendance inévitable et bien compréhensible» des équipes à rechercher des arguments favorables pour obtenir l'adhésion à leurs propres thèses.

Au contraire des missions organisées par le ministère de l'Industrie - dont on a souligné le choix controversé des participants syndicaux (17) et l'appartenance de leurs participants majoritairement à la CGC (au nombre de 29), à la CFTC (26) et en moindre mesure à la CGT-FO (15) (JoLY, 2002:189) - , la composition des missions organisées sous l'égide du ministère du Travail suit le principe d'égalité dans la représentation des syndicats ouvriers (CFTC et CGTFO) avec un nombre toujours inférieur de représentants de la CGC, critère qui est toujours scrupuleusement respecté. Le choix de participants influents et le mode de diffusion singulier des résultats apparaissent comme des indicateurs de la volonté des autorités et des dirigeants d'œuvrer pour une transformation du mouvement ouvrier.

Quant à l'organisation et aux thèmes des missions, si la direction du Travail accepte les suggestions de l'ECA et les adapte bien souvent (18), elle garde le contrôle, malgré les pressions des autorités américaines d'un côté et des syndicalistes de l'autre. Connaissant la fragilité de l'entente, elle anticipe les éventuelles critiques et introduit des modifications, de manière à ne pas heurter les organisations syndicales. Ainsi, elle empêche la réalisation d'une mission portant sur « les modalités de l'action syndicale ouvrière aux États-Unis», proposée par des représentants du patronat, et envisageant la prise de contact avec des dirigeants syndicaux américains. De même, c'est souvent à l'occasion de la publication d'articles ou de rapports de missions que le souci de ces fonctionnaires apparaît le plus manifeste, les amenant à une réécriture des textes inter-

(16) La qualification de «technique» pour caractériser un certain nombre de ces missions peut sembler discutable. Le terme "stratégique» conviendrait mieux. Le fait de s'accommoder aux critères de financement, du fait qu'il s'agit de fonds destinés à l'aide technique, est probablement à l'origine d'une telle désignation. Il est, de même, probable que celle-ci voile la portée politique de la question. Dans le cas contraire, les critiques au sein du mouvement ouvrier se trouveraient amplifiés. (17) H. Joly (2002:189) décrit ainsi le cas d'un missionnaire, représentant de la $\mathrm{CGC}$, qui a pris sa carte syndicale uniquement pour faire partie de cette mission, sans montrer par la suite aucun attachement à cette confédération.

(18) Courriers échangés entre le directeur du Travail et le secrétaire d'État aux Affaires économiques, juin 1950; mars 1951. 
médiaires (19). Des passages sont ainsi supprimés ou nuancés afin de prévenir des prises de position de nature à susciter des protestations de la part des organisations syndicales.

À la différence d'autres missions organisées par le ministère, celles destinées exclusivement aux représentants des salariés rencontrent une série d'obstacles: notamment, l'absence de candidatures spontanées et la difficulté de recruter des missionnaires «compétents » mettent en péril certaines missions qui risquent d'être annulées. Face à cette situation, la direction du Travail fait preuve de volontarisme en maintenant des missions au prix d'un contournement des critères énoncés (âge, compétence, nombre de participants), à l'exception, on l'a dit, du principe de représentation des trois confédérations qui reste premier.

D'autres problèmes concernaient l'accueil des missionnaires dans les entreprises. Certaines visites doivent ainsi être annulées, compte tenu du nombre insuffisant d'employeurs qui acceptent de les recevoir, et lorsqu'elles sont effectuées, les participants déplorent souvent les conditions dans lesquelles elles se font (simple rencontre avec un dirigeant ou limitation à un seul secteur de l'entreprise). La direction $\mathrm{du}$ Travail «minimise» et tente d'occulter dans les rapports les passages qui dénoncent ces difficultés (20). Ces circonstances sèment le doute sur la qualité des observations recueillies et, plus encore, mettent en cause le principe même d'organisation des missions : faute de pouvoir observer librement les entreprises, une partie considérable de ces voyages est consacrée à assister à des conférences. Or, tout l'intérêt de ces voyages réside dans le fait que les syndicalistes éprouvent eux-mêmes la réalité américaine plutôt que de s'en tenir à des discours. Malgré ceci, la direction du Travail s'efforce de continuer le programme.

\section{Vers une connaissance du syndicalisme américain et de ses moyens d'action}

L'objectif de ces voyages n'était pas seulement que ces syndicalistes s'initient aux méthodes et aux techniques américaines. On attendait aussi qu'ils

(19) À la lecture d'une brochure publiée par l'AFAP, J. Gouin adresse une lettre à $\mathrm{M}^{\text {lle }}$ Piguet et à $\mathrm{M}$. Lapierre, dans laquelle il fait part des conversations «officielles et officieuses» portant sur ce document, qui n'avaient pas été prises en compte au moment de la publication. Puis, dans la modification d'un texte à paraître de l'ECA, par exemple, les noms des représentants patronaux et civils ont été conservés mais les noms des responsables syndicaux ont été supprimés, pour ne pas provoquer de tensions au sein des confédérations. Note du 10 novembre 1951.

(20) C'est le cas des rapports intermédiaires dans lesquels sont ajoutés des «papillons» indiquant qu'il semble peu «prudent» de présenter en détail ces situations. adoptent un autre état d'esprit. Par lettre, le directeur du Travail prévenait les participants: «Vous aurez donc à faire cet effort intellectuel si pénible et si fécond à la fois, de dépouiller vos propres conceptions, de rejeter momentanément les fruits de votre propre expérience. Ce sera le stade de l'observation objective des faits, auquel succéderont le stade de l'appréciation, celui de la comparaison, et enfin le stade qui permettra de dégager des conclusions constructives. Je suis sûr que vous apprécierez les bienfaits de ces démarches d'esprit [...] je suis sûr que notre pays, engagé dans un effort de rénovation sans précédent, tirera le plus grand profit de votre expérience et que dès votre retour, vous aurez à cœur de faire partager votre enrichissement personnel. Je suis sûr aussi que le ministère du Travail, qui s'est voué depuis longtemps à la solution des innombrables problèmes posés par l'industrialisation, pourra compter sur votre aide et vos conseils compétents».

Quatre de ces premières missions témoignent de la volonté du ministère de développer le conseil et l'équipement des organisations syndicales à travers la sensibilisation des dirigeants syndicaux et des techniciens «avertis» aux questions techniques et au rôle joué par les experts dans le syndicalisme américain.

La première mission, appelée Intersyndicale (21), est réalisée en 1950. Ce voyage, consacré à l'étude des positions syndicales à l'égard de la productivité, vise à comprendre les raisons qui ont conduit le mouvement ouvrier à adhérer à la politique de la productivité, et même à la promouvoir(22).

L'équipe compte quinze délégués syndicaux (six CFTC, six CGT-FO, trois CGC), deux représentants du ministère et une journaliste. La moyenne d'âge des participants est de 38 ans. À l'exception des représentants de la CFTC, qui ont un niveau d'études primaires, la majorité des participants possèdent un niveau d'instruction très élevé pour l'époque: le baccalauréat ou la licence. La catégorie socioprofessionnelle la plus représentée est celle des cadres, non seulement pour la CGC, mais aussi pour la CGT-FO. Une seule femme y participe.

Leurs responsabilités syndicales témoignent de l'importance accordée par les confédérations à cette mission: les délégués de la CFTC appartiennent majoritairement à l'échelon confédéral (23) et l'on trouve parmi eux des cadres syndicaux suscep-

(21) C'est la première des missions organisées sous la tutelle du ministère du Travail et la $25^{\mathrm{e}}$ mission envoyée aux ÉtatsUnis par la France.

(22) Note justificative de la mission et préambule du rapport final de mission.

(23) L. Delsert est secrétaire fédéral adjoint des cheminots et R. Lecœur de la Fédération des syndicats chrétiens d'employés, techniciens et agents de maîtrise. Tous les deux membres du bureau confédéral. 
tibles de devenir des figures de référence (24). Les délégués de la CGT-FO sont plutôt secrétaires généraux ou membres des bureaux des fédérations (25). Enfin, les délégués de la CGC sont représentatifs de la composition de cette nouvelle organisation syndicale issue des associations d'élèves des grandes écoles (26).

Les participants retiennent l'attitude essentiellement pragmatique des syndicats américains face à la productivité. En effet, ces syndicalistes estiment que leur rôle n'est pas de s'opposer au progrès technique, par peur du chômage technologique, mais d'en limiter les effets par des mesures appropriées de reclassement des chômeurs. Pour assumer pleinement ce rôle, les syndicats doivent avoir accès à une information générale et spécifique. Les missionnaires reconnaissent a priori que cette attitude a porté ses fruits, car elle est déterminante dans la construction du climat social. Telle est la seconde conclusion tirée de ces voyages. Surpris, ils rapportent des anecdotes sur le climat social américain caractérisé par des rapports de coopération entre la direction et le personnel en ce qui concerne les questions de productivité. Des exemples «extrêmes» illustrent ce climat, allant parfois «jusqu'à des prêts d'argent du syndicat ouvrier à l'employeur». Certains rapports n'hésitent pas à affirmer la nécessité de dépasser

(24) C'est le cas de J. Vandenbussche, secrétaire général de la FGF-CFTC, de S. Troisgros, (1904-1993), secrétaire générale adjointe de la CFTC, l'une des premières femmes à accéder à la direction de cette confédération en 1948. Elle sera plus tard secrétaire adjointe de la CFDT jusqu'en 1969. Elle participe au BIT de 1950 à 1952, puis est de 1960 à 1968 membre du Conseil économique et social et, en 1970, membre de l'Unesco. Enfin, P. Michon (1912-1990), secrétaire général adjoint de la CFTC, jociste, mobilisé, emprisonné puis libéré en 1942. Il participe à un réseau de Résistance, est arrêté par la Gestapo et déporté. Après son retour en 1945, il est élu au Bureau confédéral en 1946 et 1947.

(25) Parmi eux: H. Delaplace, secrétaire de la Fédération des industries chimiques et du verre, il est élu à la commission exécutive confédérale où il siége entre 1959 et 1977 ; P. Mounicq, secrétaire général adjoint du Syndicat national des ingénieurs et cadres du textile, membre du bureau de la Fédération nationale des ingénieurs et cadres; A. Sidro (19061965), secrétaire général de la Fédération des employés et cadres de 1950 à 1965, membre de la commission exécutive, membre de la commission supérieure des conventions collectives. Il fut l'un des créateurs du journal Résistance Ouvrière et une figure de référence très active dans le syndicalisme des «cols blancs» et dans la mise en place d'institutions paritaires (Siwek-Pouydesseau, $2003: 131$ ). Il est aussi l'un des animateurs de la minorité «moderniste» de FO au cours des années 1960 avec d'autres dirigeants comme Cottave, Laval, Labi... (Yon, 2008) et, enfin, G. Thorel, membre du bureau confédéral de la Fédération nationale du papier-carton, membre délégué à la Commission nationale de conciliation des conflits du travail. Chrétien, collaborateur de la revue Esprit et l'un des animateurs du premier bulletin de la minorité, Le courrier syndical, au début des années 1950 (Mouriaux, 1987).

(26) Par exemple, J. Cailleaud, ancien élève de l'École des Mines, directeur commercial (après avoir été ingénieurconseil), président du Syndicat national des cadres de la chimie et A. Bicheron, issu de l'École nationale supérieure des industries agricoles, directeur général d'une distillerie d'alcool et membre du comité directeur de la CGC. la logique d'affrontement, estimant que l'esprit de collaboration est plus profitable que celui de lutte des classes. Ces commentaires s'adressent aussi aux employeurs qui doivent accepter l'idée de faire collaborer les représentants des ouvriers à l'étude des temps et des mouvements... Les rapports intermédiaires témoignent de la surprise des missionnaires face aux services de recherche et aux sources d'information dont disposent les syndicats américains. Les conclusions de cette mission sont largement diffusées (27), ce qui semble démontrer l'intérêt des autorités pour les pistes de réflexion engagées.

Un deuxième voyage, en 1950, résulte d'un projet antérieur au programme de productivité. En accord avec G. Walusinski (28), secrétaire général de la Fédération de l'éducation nationale (FEN, FO), P. Vignaux (29) sollicite auprès de la direction du Travail la réalisation d'un voyage portant sur l'éducation ouvrière, depuis longtemps prévu avec des syndicalistes américains. Un an plus tard, moyennant quelques adaptations, le projet est accepté sous la forme d'une mission. Un indicateur, qui peut sembler a priori secondaire, est en réalité significatif: l'intitulé de la mission est modifié. Le titre Information ouvrière et études syndicales est - à la suite d'un compromis - préféré au titre initial Éducation ouvrière, qui prêtait «à des malentendus ». Ce changement correspond à l'élargissement du thème de la mission: $P$. Vignaux écrit dans une lettre qu'il a dû intervenir «vigoureusement» auprès du cabinet du ministre car une mission consacrée seulement à l'éducation ouvrière n'était pas du goût des autorités et ne correspondait pas à l'objectif assigné aux fonds, plutôt destinés à financer une aide «technique». Les autorités américaines ne peuvent donc pas financer directement la formation des experts syndicaux, mais seulement contribuer au développement des organisations syndicales. Après remaniements, la direction propose d'intégrer dans la composition de ce groupe des membres de la CGC car désormais cette mission ne concerne plus seulement l'élément ouvrier. Cette démarche s'inscrit, comme nous l'avons déjà mentionné, dans les efforts déployés pour conserver la forme de «mission syndicale» sans exclusion des trois confédérations, en dépit des profils des candidats. On verra en effet que, parmi les dix-sept membres de cette mission (sept CFTC, sept CGT-FO, deux CGC et un chef de mission), seule la CGC ne compte pas de militants expérimentés sur les questions de formation, les autres étant en majorité des représentants syndicaux

(27) Le rapport final est publié dans Les Cahiers de la productivité $\left(\mathrm{n}^{\circ} 20-23\right)$ sous le titre «Le syndicalisme dans la vie américaine» et il est diffusé également sous forme de brochure avec un tirage largement supérieur à celui des autres missions: il est tiré à 20000 exemplaires alors que, jusqu'à l'année 1952, le tirage habituel des rapports est de 1000 exemplaires.

(28) G. Walusinski (1915-2006), agrégé de l'université, collaborateur au Centre d'éducation ouvrière.

(29) Lettre de P. Vignaux, Chambéry, le 16 juillet 1949. 
ayant œuvré activement sur les thèmes de l'éducation ouvrière et de la formation syndicale (30). Sur les quatorze représentants de la CFTC et de la CGTFO, huit sont professeurs (agrégés ou professeurs d'enseignement technique (31)). Agés de 38 ans en moyenne, ces représentants ont un niveau de formation très élevé.

Outre leur reconnaissance professionnelle, ce sont des hommes d'action et de conviction qui sauront transmettre leurs idées au sein du mouvement syndical mais pas immédiatement. En effet, , aux yeux des fonctionnaires de la direction du Travail, les parcours de ces participants pouvaient a priori laisser supposer qu'ils allaient proposer des changements au sein de leurs structures. Or, il n'en sera pas ainsi: lors du dernier rapport de quinzaine, le chef de mission écrit à $\mathrm{J}$. Gouin que les participants sont des «spécialistes de l'éducation ouvrière mais pas des dirigeants syndicaux [...]. Ils ne s'estiment donc pas qualifiés pour "prendre position" au nom de leurs organisations et veulent consulter cellesci avant de le faire»(32). Le responsable regrette l'absence de suggestions constructives en vue d'une amélioration possible de la situation économique en France. En fait, la raison invoquée par les membres de la mission semble constituer un prétexte pour ne pas se prononcer sur des thèmes controversés au sein des confédérations. Une fois de plus, l'on voit le caractère volontariste des actions de la direction du Travail à l'intention de syndicalistes qui restent encore à convaincre.

Il ressort de cette mission que le contenu des programmes de formation et des études entreprises par les syndicats est déterminé par le souhait de leurs dirigeants de «participer aux affaires du pays». Les

(30) Citons les représentants de la CFTC: R. Lebescond, secrétaire confédéral, responsable national du Service de l'éducation ouvrière et fondateur de l'Institut de culture ouvrière, il participe, plus tard, à la commission de réflexion et de proposition sur les valeurs de la CFDT, puis deviendra membre du Conseil économique et social; J. Rupert, secrétaire général, responsable de l'éducation ouvrière, il devient responsable de la formation au sein de la CFDT; B. Vacheret (1911-1991), il organise en 1936 le mouvement d'éducation ouvrière, est membre du bureau national du SGEN et, participe au groupe Reconstruction; M. Singer, secrétaire régionale du SGEN et aussi F. Girod, Comité national du SGEN, tous les deux militants dans les mouvements d'éducation ouvrière. Parmi les délégués de FO: R. Étienne (1897-1974), secrétaire confédérale FO, chargée de l'éducation ouvrière; D. Wurmser, directrice d'études au Centre d'éducation ouvrière, membre du bureau de la Fédération internationale des associations d'éducation ouvrière, responsable de la délégation, R. Hagnauer (19011986), secrétaire adjoint de la FEN, secrétaire de la commission éducation, animateur de la revue La révolution prolétarienne ainsi que collaborateur au Cri du peuple et à L'école Libératrice. Enfin, G. Walunsinski compose aussi l'équipe de missionnaires.

(31) Notamment Raymond Calvel (1913-2005), secrétaire adjoint du syndicat du personnel de l'enseignement technique (FEN-FO).

(32) Lettre du chef de mission à J. Gouin, le 28 septembre 1950. thèmes proposés sont davantage voués à la résolution des problèmes pratiques qu'aux questions théoriques, telles que la politique interne, la négociation des conventions collectives, la conduite de négociations, l'étude des temps et mouvements, le rôle et l'action du délégué, le droit ouvrier et l'histoire du mouvement syndical. De leur côté, les participants soulignent, en confirmant les résultats de la première mission, l'intérêt de former des techniciens de l'organisation industrielle au sein des syndicats.

Suivent, dans la liste des voyages réservés aux syndicalistes, une mission consacrée à l'étude de l'organisation et du fonctionnement des services des syndicats (1951), et une autre à l'étude de la pratique des conventions collectives (1951), puis en 1952, la mission Services de documentation et de recherches des syndicats. Cette dernière est organisée à l'initiative du Labor division qui propose le programme, la documentation et le nombre de participants (33). Sa justification réside dans le fait que les services de documentation et de recherche des syndicats américains, tout en étudiant les questions relatives à la productivité, contrôlent les normes et l'organisation du travail dans les entreprises et exercent un rôle dans l'adhésion des travailleurs à cette politique. C'est pourquoi il semble utile que du personnel des syndicats français scrute ces organismes. Les confédérations françaises ont d'ailleurs, au retour de la première mission, engagé une réflexion sur la possibilité de créer leurs propres bureaux d'études (34).

L'objet de cette mission est très spécifique. Le directeur du Travail reconnaît même qu'il peut apparaître «étroit», tout en le justifiant: «Vous constaterez, une fois aux États-Unis, qu'il n'en est rien et qu'en concentrant votre attention sur les services d'études et de recherches des syndicats, vous allez au cour même de la puissance du mouvement ouvrier américain [souligné dans le texte de l'allocution]. Rien n'est plus révélateur des principes et des moyens d'action de ces organisations que leurs états-majors d'ingénieurs, d'économistes, de sociologues formés dans les mêmes écoles, de valeur aussi grande que les techniciens patronaux. Rien aussi ne traduit mieux l'évolution profonde et nécessaire de la politique et des méthodes syndicales qu'entraîne actuellement, dans tous les pays industriels, l'accélération du progrès technique» (35).

(33) Lettre du directeur du Travail, datée du 26 mai 1951, ainsi que la lettre de la CGT-FO du 11 juillet 1951, dans laquelle il est mentionné que le Labor Division demande au ministère d'envoyer deux missions pour les services de recherches des syndicats français.

(34) Comme l'attestent les lettres échangées entre R. Richard, J. Gouin et E. Lambert datés de juin et juillet 1951, ainsi que les débats au sein des congrès confédéraux de la CGT-FO ( $c f$. IHS, Nanterre).

(35) Note de la direction du Travail, à propos de l'allocution du directeur aux membres de cette mission. 
Treize membres composent cette mission: un chef de mission, douze représentants syndicaux (deux CGC, cinq CFTC et cinq CGT-FO). Tous exercent une activité professionnelle dans de grandes entreprises privées (Rhône-Poulenc, Shell) ou publiques (Électricité et Gaz de France, RATP, SNCF, Renault). Ils possèdent pour la plupart d'entre eux un niveau de formation très élevé, trois étant issus de grandes écoles de commerce (36), trois étant titulaires d'un doctorat en droit (37) et un autre titulaire d'une licence en droit et sciences politiques. Contrairement à d'autres missions, ces membres sont d'âges très divers. Ils appartiennent à des générations différentes qui possèdent des expériences non moins diverses: deux des représentants, vraisemblablement tout juste sortis de leurs études, déclarent n'avoir jamais travaillé, tandis que d'autres ont déjà accumulé une longue expérience professionnelle et militante(38). À l'exception de cinq de ces missionnaires (39), les autres semblent, davantage que des dirigeants de premier rang, des militants formés et sensibilisés aux questions techniques, susceptibles de devenir des «techniciens appointés» du syndicalisme, selon la formule utilisée à l'époque.

Ces participants découvrent l'importance des ressources documentaires et admirent particulièrement la qualité des recherches et de l'information des syndicats américains. Ils sont frappés par le nombre de spécialistes, leur armature administrative et financière, l'importance de leurs services d'éducation et de documentation. Les techniciens qui y travaillent sont des militants ou, plus fréquemment, des collaborateurs recrutés parmi les universitaires. On trouve parmi eux des ingénieurs, des fabricants spécialisés, des économistes, des métreurs, des bibliothécaires. Ces bureaux de recherche sont très hétérogènes et leur organisa-

(36) M. Gramblin (CGC), directeur de Rhone-Poulenc, et J.J. Simonneau, né en 1926, n'ayant jamais travaillé avant son poste de secrétaire (CGC).

(37) Il s'agit des trois délégués CGT-FO: A. Spire, administrateur civil au ministère du Travail; R. Nayrolles, secrétaire du Comité interfédéral des fonctionnaires et postiers et R. Rul, responsable des services d'études économiques.

(38) C'est ce qui contraste, par exemple, avec le parcours de R. Rul qui, avant la mission, avait été instituteur, militant du Parti socialiste, secrétaire fédéral adjoint de la ville d'Ajaccio pour le Parti socialiste et cosecrétaire de la Fédération socialiste de la Seine. Il avait été mobilisé pendant la guerre et s'était occupé du service de documentation de la CGT puis de la CGTFO à la suite de la scission.

(39) Parmi eux: J. Bon, président du syndicat CFTC des employés techniciens du caoutchouc; C. Glatiny, secrétaire CGT-FO du syndicat du ministère des Anciens combattants; P. Butet, jociste, résistant et déporté qui devient secrétaire CFTC de la Fédération des cheminots et membre du bureau confédéral de la CFDT, ainsi que J.-P. Murcier conseiller juridique, puis membre du bureau d'études de la CFTC-CFDT et enfin, secrétaire confédéral. Il s'attache depuis le début des années 1950 à revendiquer le rôle de conseiller, particulièrement juridique, auprès des syndicats et contribue à légitimer ce rôle au sein de sa confédération (WILLEMEZ, 2003/4: 30). tion varie en fonction de la taille de la structure et de l'échelon syndical. Certains s'occupent de questions purement économiques, de caractère national, susceptibles d'alimenter la discussion avec le gouvernement ou de compléter l'information des fédérations. Consacrés aussi bien aux questions professionnelles qu'économiques, ils font penser à de véritables appareils. C'est le cas de l'un des services étudiés, qui comporte cinq équipes techniques, chacune correspondant à une spécialisation en fonction des questions traitées: une équipe prépare les discussions préalables aux négociations des conventions collectives, une autre organise la documentation économique sur l'industrie ou les différentes entreprises, une troisième s'occupe de l'action sur le plan législatif et politique, une quatrième est plus spécialisée sur le plan communautaire, enfin, une dernière produit des études de mouvements, des calculs de temps... Ces services effectuent également des recherches à la demande du syndicat. Sont ainsi mentionnés des services qui se développent en fonction de la nature des revendications ouvrières, par exemple le développement d'une job evaluation dans un secteur où ont été constatés des abus patronaux en matière de détermination des temps et mouvements. Les missionnaires relèvent également la création d'un service consacré aux questions sociales dans une fédération qui compte une importante population d'ouvriers défavorisés.

L'importance accordée aux bureaux syndicaux est confirmée par la réalisation, plus tard, d'autres missions consacrées à l'analyse de ces organismes: des groupes de syndicalistes partiront étudier les bureaux de recherche des syndicats américains de la métallurgie et de la construction mécanique (1955), de la confection (1955) ainsi que des industries chimiques et pétrolières (1955).

Une quatrième mission, La comptabilité au service de la productivité et des syndicats, appelée aussi Comptables industriels syndicaux (1953), s'inscrit dans la même veine. Elle reflète une autre préoccupation de l'époque, celle d'instaurer un contrôle rationnel des organisations. Depuis 1946, les élus des comités d'entreprise peuvent faire appel à un expert-comptable, mais ce recours n'est guère utilisé. L'objectif du voyage est d'envoyer outreAtlantique des comptables industriels qui, à leur retour, puissent exercer les fonctions de conseillers techniques auprès des représentants des salariés.

Le groupe comprend douze représentants des syndicats (cinq CGT-FO, quatre CFTC, trois CGC) et un chef de mission représentant le ministère. Cette composition est plus homogène que les précédentes, la majorité des membres étant des militants 
de «deuxième rang», et sont tous comptables (40). Ce choix semble répondre à un souci d'efficacité, tel que l'avait exprimé quelques années auparavant un militant CGT-FO: ces missionnaires devaient pouvoir «jouer leur rôle avec plus de dynamisme à leur retour» (41). Ils sont censés pouvoir constater, d'une part, comment la comptabilité aide à l'accroissement de la productivité et, d'autre part, comment la maîtrise des renseignements financiers sur les entreprises conduit les organisations syndicales à mieux négocier les conventions collectives (42).

Le rôle d'un type particulier de spécialiste au sein du syndicat, le technicien comptable, est donc mis en évidence. Au regard de la situation française, ces comptables sont d'abord considérés comme une garantie d'efficacité dans l'action syndicale. Ils permettent en effet aux élus d'étayer leurs arguments, et non pas seulement d'être «livrés à leur propre intuition». Grâce à cet appui, l'élu responsable se trouve en mesure de réagir face à un problème de productivité, de calcul de normes, d'évaluation du temps du travail, de discussions sur les prix de revient et il ne se laisse pas submerger par les chiffres avancés par les techniciens patronaux. Ces responsables jouent ainsi un rôle capital dans les discussions et les négociations. Les missionnaires considèrent que les syndicalistes français n'ont pas ces capacités. Leurs témoignages pointent l'insuffisance de l'appui fourni aux militants par les confédérations. Ils citent des cas où les délégués siégeant «seuls», sont obligés de faire appel à des techniciens privés lorsqu'ils rencontrent des difficultés. C'est pourquoi les participants préconisent la formation de techniciens syndicaux «aptes à parler chiffres, apportant ainsi dans la discussion un sérieux appoint»(43). C'est en cela que les missionnaires voient la marque d'un syndicalisme «moderne», «tel que nous l'avons vu aux ÉtatsUnis», qui est aussi l'une des conditions du succès des négociations.

À la fin des quatre missions mentionnées, et pour garantir que soit réalisé le travail de réflexion, de comparaison et de mise en forme, le directeur du Travail invite tous les missionnaires à un

(40) Pour la CGT-FO, J.-P. Gaillard, chef du groupe comptabilité EDF-GDF, et P. Raynaud, directeur du personnel et contrôleur général de la comptabilité du centre hospitalier de Chartres. Pour la CFTC, M. Georges, chef de comptabilité industrielle, SA Saints Frères à Leers; R. Housseau, chef comptable adjoint, Charbonnière de Paris; R. Lepert, chef de la section comptabilité, Poliet et Chausson, et F. Raymond, comptable général usine de moutiers. Enfin, pour la CCG, P. Chupin, chef de la comptabilité industrielle, société alsacienne de constructions mécaniques et L. Morelet, chef de la comptabilité, Alsthom.

(41) Congrès Confédéral CGT-FO, Paris, octobre 1950.

(42) Rapport intermédiaire de mission, mai 1953.

(43) Rapport de cette mission non publié réalisé par un syndicaliste FO, M. Cuvillier. congrès (44). Ce rassemblement, permet non seulement de réfléchir collectivement aux enjeux de l'expérience réalisée et de pérenniser les liens établis lors des voyages, mais constitue aussi un moyen de pallier la difficulté provoquée par la dispersion professionnelle et géographique des syndicalistes, qui, une fois de retour, parviennent difficilement à se réunir pour rédiger le rapport final. À travers cette action, la direction aide les chefs de missions qui se plaignent de ne pas recevoir de contributions aux rapports de la part des participants.

\section{La création des bureaux techniques des syndicats français}

De ces quatre voyages se dégage l'intérêt d'une «fonction technique» pouvant être remplie par les syndicats au sein des entreprises. Désormais, l'intention des fonctionnaires est de développer cette fonction de mesure et d'évaluation technique effectuée au service des organisations syndicales, qui est perçue comme un levier permettant de renforcer la légitimité syndicale sur de nouvelles bases et privilégiant un esprit de négociation et de responsabilité. De par leur fonction de «traduction», de traitement et de production de l'information, les techniciens permettent aux syndicalistes de devenir de véritables interlocuteurs du patronat et d'intensifier la conclusion des conventions collectives. Comme se le demande SAUERWEIN (1953: 5), "la méfiance de la classe ouvrière n'aurait-elle pas pour origine l'incapacité dans laquelle elle se trouve de porter un jugement valable sur la gestion des entreprises? On lui oppose traditionnellement son inexpérience des problèmes financiers, mais a-t-on fait jusqu'ici des efforts suffisants pour les lui faire assimiler?».

Depuis la fin des années 1940, d'autres avaient soulevé ce rôle particulier des techniciens syndicaux américains qui dénote une «attitude positive» : «Conseillés par des ingénieurs en organisation industrielle, les Trade Unions ont introduit des clauses de productivité dans de nombreuses conventions collectives. Ces techniciens ont réussi à dissiper toutes les craintes des syndicats » (45). Or, il a fallu attendre le déroulement des missions décrites pour que les syndicalistes s'emparent enfin de la question. Ce sont, en effet, les missionnaires, à leur retour, qui se chargent d'affirmer la nécessité de former des techniciens, spécialistes des questions sociales et scientifiques au sein de leurs orga-

(44) Plus précisément, il s'agit d'une invitation à un congrès de syndicalistes français participant aux missions. Courrier d'E. Lambert aux chefs de missions, 1952.

(45) «Les syndicats ouvriers, les comités d'entreprise et l'accroissement de la productivité », L'aide américaine à la France. Bulletin publiant des informations relatives au Plan Marshall, bimensuel, $\mathrm{n}^{\circ} 20$, avril 1949. 
nisations (46). Quoique eux-mêmes conscients de l'impossibilité d'opérer un transfert des méthodes américaines, ils s'accordent sur l'intérêt de s'inspirer de ces initiatives.

Sous l'impulsion de ces expériences et avec l'appui de la direction du Travail, ils mettent en place des dispositifs d'études mais se heurtent d'emblée à des réticences au sein des organisations syndicales, et le climat de confiance espéré peine à s'instaurer. Ainsi, dès le début, ils expriment leur crainte de voir des techniciens prendre une place trop importante au sein des syndicats, ce qui amènerait «à faire de plus en plus confiance aux méthodes rationnelles d'organisation et à se défier des arguments "émotionnels" ", et ainsi à perdre, dans une certaine mesure, le contact avec la base. Ces missionnaires considèrent que ce problème est propre à la France et qu'ils doivent impérativement le prendre en compte si l'on veut atteindre des résultats identiques à ceux des États-Unis. Mais les adaptations tentées ne se limitent pas simplement à l'intégration d'un nouveau moyen d'action, elles impliquent surtout une évolution radicale de la manière d'appréhender l'intervention des organisations syndicales.

Bien qu'éphémères, ces adaptations permettent de comprendre à quel point les changements introduits sont, au fond, seulement souhaités par ces fonctionnaires et par certains cadres syndicaux. L'histoire des «bureaux intersyndicaux de recherche sur la productivité» illustrent la complexité de la tâche entreprise. Créés à la suite de la première mission intersyndicale, composés de techniciens et dirigés par des responsables syndicaux des trois confédérations libres, ils ont pour rôle de capter l'intérêt du mouvement ouvrier et de devenir de véritables organismes d'appui technique des militants. Ces bureaux assurent un contrôle technique des mesures de productivité favorable à une action conjointe des syndicats libres. Soutenus professionnellement et financièrement par le ministère (grâce aux fonds américains), ils proposent un regard technique, présenté comme nécessaire à la négociation grâce à la constitution de revendications «crédibles».

Le Centre intersyndical d'études et de recherche de productivité (CIERP, 1951) (Boulat, 2008), le premier d'entre eux, ainsi que d'autres qui lui succèdent (le Bureau intersyndical d'études de l'industrie cotonnière, BIEIC, 1953, qui deviendra plus tard le Bureau intersyndical d'études de l'industrie textile, BIEIT, 1955), interviennent à la demande des syndicats. Avec l'accord des employeurs, les experts ou techniciens procèdent à l'analyse et au contrôle des postes de travail et réalisent des études économiques

(46) L. Rey rend compte d'une réunion entre plusieurs syndicalistes FO participant à des missions différentes, Rapports France-États Unis, ${ }^{\circ}$ 46, 1951, pp. 52-56. pour dispenser des conseils techniques aux sections syndicales.

Leur activité consiste à produire des études économiques généralement motivées par les changements survenus dans l'organisation de la production. À travers un rapport, les techniciens évaluent si l'effort demandé aux salariés est compatible avec un niveau «normal» de travail et fournissent des éléments objectifs pouvant alimenter convenablement les négociations relatives à la rémunération. Ces centres organisent également des sessions de formation destinées aux ouvriers et syndicalistes des différentes branches. Pour en favoriser le rayonnement, la direction du Travail veille à ce qu'un membre des bureaux participe aux missions sociales. Il favorise également la diffusion des travaux des techniciens. C'est là une manière de contribuer à légitimer leur place au sein des confédérations syndicales françaises. Le leitmotiv pour justifier leur action est le suivant: «Plutôt que de se laisser imposer des solutions auxquelles les organisations syndicales seraient étrangères, mieux vaut encore participer aux discussions et s'efforcer de faire comprendre la raison des réticences des salariés envers la productivité et la raison de leur adhésion: la productivité doit servir à élever le niveau de vie des salariés » (47).

Ces institutions se heurtent, dès l'origine, à l'indifférence du mouvement syndical. Très tôt (1953), une note confidentielle émanant de la direction du Travail déplore le risque de "cassure» du CIERP. Pour y remédier, un "Schéma d'organisation des services d'études des organisations syndicales» est suggéré. Il est même envisagé de supprimer le terme «productivité» du nom des bureaux, afin de les faire mieux accepter(48). De plus, ces bureaux cherchent à élargir leur sphère d'action: ils envisagent d'aller au-delà des études sur la productivité dans l'entreprise en proposant des études au plan régional. Ils restent cependant très peu sollicités: un rapport(49) considère que le CIERP réalise des études «mineures faute de demandes d'envergure». Le désaccord de J. Gouin est manifeste, il note: «Pas certain que leur travail soit insuffisant», «pas sûr qu'ils ne soient pas assez sollicités», «trop catégorique» ou «a connu plus de succès que de mécomptes ». C'est là une manière de continuer à soutenir l'action de ce bureau. Un autre rapport est plus nuancé: il dénombre seulement soixante-seize

(47) Annonce du congrès des travailleurs envoyés en mission, organisé par le CIERP dans Les Cahiers de la productivité $\left(\mathrm{n}^{\circ} 7\right.$, 1952). Une note dans le numéro suivant indique que le congrès s'est tenu et que «c'est peut-être l'un des événements les plus importants de ces dernières décennies dans l'ordre économique et social», Les cahiers de la productivité $\left(\mathrm{n}^{\circ} 8,1952\right)$.

(48) Note confidentielle de la direction du Travail du 30 avril 1953.

(49) Rapport réalisé à la demande du commissaire général à la productivité en 1957 et rédigé par J. Ploquin. 
interventions réalisées par le BIEIT entre 1953 et 1957. Si ces interventions sont peu significatives en nombre, leur contribution au règlement des conflits est pourtant largement confirmée par les faits: quarante-cinq interventions se sont conclues par des avantages pour le personnel. De plus, le bilan indique que lorsque les rapports ne justifiaient pas les revendications du personnel, la prise en compte de ces informations a évité des conflits, et c'est logiquement cette donnée qui sera retenue par la direction du Travail. Malgré ces bilans mitigés, la volonté de développer ce moyen d'action est certainement à l'origine d'un financement qui se prolongera jusqu'à la fin des années soixante, et même de la proposition d'ouvrir une ligne de crédit pour les syndicats et les branches, exclusivement destinée à la venue de conférenciers de ces bureaux.

Toutefois, l'histoire des bureaux est rythmée par une série de contestations du côté des militants, méfiants à l'égard des thèmes de recherche et des méthodes qui peuvent toucher à la cogestion avec le patronat. De vives réactions ont lieu au sein de la CFTC (50) au moment de leur mise en place et d'âpres conflits internes se soldent par le retrait de la CGT-FO de ces centres (51). À l'instar d'autres innovations proposées au sein des entreprises qui rencontrent des résistances dans leur application locale (KUISEL, 1996: 164), ces difficultés mettent en évidence le caractère insuffisamment consensuel de ces dispositifs. Elles témoignent ainsi, dans le cas des bureaux d'études syndicaux, de la difficulté pour les autorités politiques et les directions syndicales de convaincre leurs adhérents du bien-fondé $\mathrm{du}$ recours aux techniciens et aux experts. Une tentative de dernier recours proposée par la direction tend à ce que ces bureaux deviennent des organismes financés par les confédérations mêmes et non par les organismes officiels. L'objectif est que les militants les considèrent comme «leur» mandataire, afin de dissiper la méfiance suscitée, mais cette tentative ne semble pas avoir été plus efficace. Des centres d'études propres à chaque organisation syndicale voient le jour quelques années plus tard: le Bureau d'études économiques et sociales (BEES) de la CGT-FO en 1956, le Bureau technique de la CGC en 1957 et le Bureau de recherches et d'action économique (BRAEC) de la CFTC en 1957. Tous trois sont des associations loi 1901 et sont contraints de solliciter des subventions du Fonds national de

(50) Des conflits surviennent au sein de la Fédération du textile (ADAM, op. cit., p. 256) et des protestations émanent de délégués de la métallurgie, du bois et de la chimie lors du congrès confédéral de la CFTC (Boulat, 2006: 455).

(51) C'est lors du congrès national que s'est produit le retrait de FO du CIERP et la mise en cause de son directeur et représentant par FO, R. Richard. Même si ce dernier explique dans un article (RICHARD, 1953) qu'il ne s'agissait pas d'une opposition au programme de productivité, les déclarations d'autres responsables syndicaux de cette organisation permettent de relativiser son point de vue. la productivité. Lors de leur création, la direction du Travail veille à ce que l'activité de ces institutions ne court-circuite pas l'activité des bureaux intersyndicaux, en définissant leurs objectifs et leurs domaines d'intervention(52). L'exposé des motifs justifiant la naissance de ces institutions indique que «de nombreuses centrales syndicales disposent à l'étranger de bureaux d'études qui facilitent la discussion, la négociation et la conclusion d'accords ou conventions. Il est communément admis que le mouvement syndical doit disposer de bureaux d'études qui assaillent quotidiennement ses militants pour lui permettre de proposer des solutions techniques aux nombreux problèmes. Les syndicats ont besoin d'une aide qui doit pouvoir éclairer leurs délibérations et les décisions qu'ils ont à prendre» (53). Ces institutions ne se consacrent cependant pas uniquement aux études et aux mesures de productivité et leur activité s'oriente progressivement vers d'autres: formation, think tank, études de conjoncture...

En ce qui concerne les bureaux intersyndicaux, leur faible légitimité aux yeux des militants, d'une part, et le fait que l'organisation syndicale majoritaire ne participe pas à ce type d'activité, d'autre part, les prive d'une véritable assise. Ils ne réussiront donc pas à perdurer, du moins sous leur forme initiale. Ces institutions ont cependant contribué à définir une conception de la défense des salariés centrée sur une connaissance technique et une pratique de la négociation plutôt que sur l'affrontement.

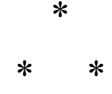

Si les rapports de missions, publiés notamment par l'Association française pour l'accroissement de la productivité (AFAP), ne constituent pas une source fiable pour analyser le point de vue des participants, du fait de leur caractère propagandiste (KuISEL, 1988), les rapports non publiés, les lettres et les conférences rendent compte plus fidèlement des intentions et des actions impulsées par les autorités politiques. Ainsi a été décrite l'intervention d'un département du ministère du Travail qui, convaincu de la faiblesse technique des organisations syndicales, a saisi l'occasion d'y remédier par le biais des missions. Ces fonctionnaires sont persuadés que celles-ci représentent une opportunité pour la France car la compétence technique tant sollicitée lors des

(52) Ils vont intervenir au niveau régional et national et les bureaux intersyndicaux sont chargés des expertises techniques au niveau des entreprises. $C f$. la note confidentielle de J. Gouin à B. Piguet du 25 octobre 1955 et la lettre d'A. Malterre à J. Gouin du 28 novembre 1956.

(53) Lettre d'O. Raffalovich et E. Lambert, portant sur le projet de création d'un bureau d'études de la CGT-FO, le 14 décembre 1955. 
missions est un moyen d'appeler à un changement symbolique et politique du rôle des syndicats: en s'informant, en se formant, en comprenant les questions économiques et techniques, les syndicats se préparent à assumer un rôle «à la fois revendicatif et constructif» (RICHARD, 1953 : 304).

La persévérance dans l'organisation des missions syndicales et les controverses nées à l'occasion du développement d'une fonction technique syndicale témoignent, tout comme la sous-utilisation des spécialistes mis au service des militants, de la difficulté à ancrer ce type de pratiques dans l'univers des représentations et des doctrines d'action du mouvement ouvrier français de l'époque. À court terme, les résultats modestes des actions engagées au retour des missions contrastent avec l'important effort déployé par l'élite moderniste initiatrice de ce mouvement. Les actions et les institutions décrites ont montré les limites d'une tentative: celle de faire évoluer les acteurs syndicaux du conflit vers le dialogue. Cependant, elles ont fixé les prémisses d'une évolution qui interviendra une vingtaine d'années plus tard, à la faveur d'une nouvelle formulation du problème en termes de droit à l'information des salariés. C'est seulement alors qu'une activité d'expertise syndicale professionnelle réussira à s'imposer au sein des relations professionnelles, en empruntant aux méthodes inspirées de l'expérience américaine.

\section{Bibliographie}

Adam G. (1964), La CFTC, Paris, Armand Colin.

BARJot D. et al. (2002), Catching up with America. Productivity missions and the diffusion of American Economic and technological influence after the Second World War, Presses de l'université de Paris-Sorbonne.

Béthouart B. (1999), Des syndicalistes chrétiens en politique (1944-1962), Paris, Presses universitaires du Septentrion.

BÉthouart B. (2006), Le ministère du Travail et de la Sécurité sociale de la libération au début de la Ve République, Presses universitaires de Rennes.

Boltanski L. (1981), «America, America... le plan Marshall et l'importation du management», Actes de la recherche en sciences sociales, $\mathrm{n}^{\circ} 38$, pp. 19-41.

Boltanski L. (1982), Les cadres, la formation d'un groupe social, Paris, Les Éditions de Minuit.

Boulat R. (2002), «Le concept de productivité en France de la Première Guerre mondiale aux années soixante», Travail et Emploi, n ${ }^{\circ}$ 91, pp. 43-56.

Boulat R. (2006), Fourastié, la productivité et la modernisation de la France (années trente-années cinquante), deux tomes, thèse d'histoire, université de Franche-Comté, décembre.

Boulat R. (2008), Jean Fourastié, un expert en productivité. La modernisation de la France (années trente-années cinquante), Paris, PUF.

Chatriot A. (2006), «L'introuvable démocratie sociale: débats intellectuels, luttes politiques, acteurs sociaux et administratifs, 1944-1950 », in Chatriot A., Join-Lambert O., Viet V. (sous la direction de), Les politiques $d u$ Travail (1906-2006): acteurs, institutions, réseaux, Presses universitaires de Rennes, pp. 385-401.

Crouzet F. (2002), «Conclusions», in Barjot D. et al., Catching up with America. Productivity missions and the diffusion of American Economic and technological influence after the Second World War, Presses de l'université de Paris-Sorbonne, pp. 427-438.
Fourastié J., Teper M., Kaplan H., Christa R., et al. (1952), La productivité des idées et des réalisations, Paris, La Fédération.

Godson R. (1976), American Labor and European Politics. The AFL as a Transnational Force, éd. Crane, New York, Russak \& Company.

Guigueno V. (2002), "What they saw, what they wrote, what we read: the American experience in the reports of French Marshall - Plan Missionaries", in Barjot D et al., Catching up with America. Productivity missions and the diffusion of American Economic and technological influence after the Second World War, Presses de l'université de Paris-Sorbonne, pp. 197-206.

JeAnPIERre L. (2000), «Paul Vignaux, inspirateur de la "deuxième gauche": récits d'un exil français aux ÉtatsUnis pendant la Seconde Guerre mondiale», Matériaux pour l'histoire de notre temps, $\mathrm{n}^{\circ} 60, \mathrm{pp} .48-56$.

Joly H. (2002), "Sociology of the members of the French productivity missions to the USA", in Barjot D. et al., Catching up with America. Productivity missions and the diffusion of American Economic and technological influence after the Second World War, Presses de l'université de Paris-Sorbonne, Paris, pp. 183-196.

Kuisel R. (1988), «L'American Way of life et les missions françaises de productivité», Vingtième siècle, Revue d'histoire, $\mathrm{n}^{\circ} 17$, pp. 21-38.

Kuisel R. (1996), Le miroir américain, cinquante ans de regard français sur l'Amérique, Paris, J.-C. Lattès.

Maclouf P. (2007) «Fonctionnaires au Travail: bureaucratie et personnalité au ministère du Travail dans la période de son cinquantenaire», Travail et Emploi, $\mathrm{n}^{\circ} 110$, pp. 47-74.

MCGLAde J. (1995), The illusion of consensus : american business, cold war and the industrial recovery of western europe, 1948-1958, Ph-D Dissertation, Georges Washington University.

Mcglade J. (2002), “The US Technical Assistance Pro- 
gram: From revolutionnary vision to production drive", in Barjot D. et al., Catching up with America. Productivity missions and the diffusion of American Economic and technological influence after the Second World War, Presses de l'université de Paris-Sorbonne, pp. 67-86.

Mouriaux R. (1987), «Entretien avec Georges Rino», in Collovald A., Mouriaux R., Sept. entretiens avec des militants sur les relations entre Pierre Mendès-France et le mouvement syndical, Paris, FNSP-CEVIFOP.

RichARD R. (1953), «Le syndicalisme français devant les problèmes de la productivité», Revue internationale $d u$ travail, volume LXVIII, $\mathrm{n}^{\circ}$ 3, septembre, pp. 297-323.

SAuERweIN G. (1953), «L'information du travailleur, condition de la meilleure marche de l'entreprise», Productivité française, $\mathrm{n}^{\circ} 14$, pp. 5-6.

Siwek-Pouydesseau J. (2003), «Les cols blancs, fonctionnaires et employés, dans la CGT-FO», in Dreyfus M., Gautron G. et Robert J.-L., La naissance de Force Ouvrière, Paris, PUF.
TANGUY L. (2006), «Institutionnalisation de la formation syndicale et politique sociale du ministère du Travail 1950-1970», communication au colloque: Élaborations et mises en oeuvre des politiques du travail: le ministère du Travail et la société française au $\mathrm{XX}^{\mathrm{e}}$ siècle, 18-19 mai 2006.

TANGuY L. (2008), «Retour sur l'histoire de la sociologie du travail en France: place et rôle de l'ISST», Revue française de sociologie, à paraître.

Wall I. (1989), L'influence américaine sur la politique française 1945-1954, Paris, éditions Balland.

Willemez L. (2003/4), «Quand les syndicats se saisissent $\mathrm{du}$ droit: invention et redéfinition d'un rôle», Sociétés contemporaines, $\mathrm{n}^{\circ} 52$, pp. 17-38.

YoN K. (2008), Retour sur les rapports entre syndicalisme et politique: le cas de la CGT-FO. Éléments pour la sociologie d'un "monde de pensée », thèse de science politique, université de Paris I. 